\title{
Ali Akbar Khan, le maître du sarod Indien
}

\section{Huib Schippers}

Traducteur : Isabelle Schulte-Tenckhoff

\section{OpenEdition}

\section{Journals}

Édition électronique

URL : http://journals.openedition.org/ethnomusicologie/2446

ISSN : 2235-7688

\section{Éditeur}

ADEM - Ateliers d'ethnomusicologie

\section{Édition imprimée}

Date de publication : 1 janvier 1992

Pagination : 258-276

ISBN : 978-2-8257-0456-1

ISSN : 1662-372X

\section{Référence électronique}

Huib Schippers, "Ali Akbar Khan, le maître du sarod Indien », Cahiers d'ethnomusicologie [En ligne], 5 | 1992, mis en ligne le 15 décembre 2011, consulté le 19 avril 2019. URL : http://

journals.openedition.org/ethnomusicologie/2446 


\section{ENTRETIEN}

\section{ALI AKBAR KHAN, LE MAÎTRE DU SAROD INDIEN*}

\section{Huib Schippers}

En 1936, un jeune joueur de sarod fit ses débuts à la All India Music Conference de Allahabad. Ce premier concert en solo d'Ali Akbar Khan marqua le début d'une phase importante de l'histoire de la musique instrumentale indienne. Jusque là, aucun joueur de sarod n'avait su atteindre pareille maitrise technique et artistique.

Ce fut le début d'une carrière remarquable, couvrant maintenant plus de cinq décennies. Des milliers de concerts et d'émissions de radio, ainsi que de nombreux enregistrements commerciaux ont affirmé la renommée du khansahib comme le digne dépositaire de la gharāna de son père Baba Allauddin, et comme l'un des meilleurs musiciens du vingtième siècle.

Mais l'importance d'Ali Akbar Khan va au delà de ses qualités artistiques. Sa vie reflète les changements sociaux et musicaux que l'Inde a connus tout au long de ce siècle: le déclin des cours royales, le rôle de la All India Radio comme nouvelle protectrice de la musique classique, l'avènement du concert de masse, le développement de la musique enregistrée et la propagation de la musique indienne en Occident.

Ali Akbar Khan a joué un rôle crucial dans l'ensemble de ces changements: comme musicien à la cour de Jodhpur, comme producteur musical et artiste à la All India Radio de Lucknow, comme directeur de musique de films à Bombay et à Calcutta, comme sarodiste de concert acclamé à travers toute l'Inde, comme pionnier de la musique indienne en Occident, enfin, comme professeur de musique en Inde et aux États-Unis où il fonda le Ali Akbar College of Music.

Traduit de l'anglais par Isabelle Schulte-Tenckhoff. 
Peu de musiciens indiens ont été à ce point vénérés de leur vivant. Alliant une connaissance profonde de la tradition à un génie créateur, Ali Akbar Khansaheb est devenu le musicien des musiciens, tout en restant un instrumentiste hautement apprécié du public.

Au cours de sa vie, Ali Akbar Khan reçut de nombreuses distinctions honorifiques. Quand il était encore jeune, il fut nommé ustad (maître) à la cour de Jodhpur; plus tard, il reçut divers titres honorifiques du gouvernement indien, dont celui de Padma Bhûshan. Peu avant son soixante-dixième anniversaire, il fut honoré par l'Amérique qui lui attribua le prestigieux subside de la Fondation MacArthur. Le titre qu'il valorise plus que tous les autres lui fut décrété par son père qui le nomma un jour Swara Samrat (litt. «empereur du son»).

L'entretien qui suit fut compilé à partir de plusieurs interviews avec Ali Akbar Khansaheb, conduits en vue d'établir sa biographie, à paraître sous le titre Swara Samrat-Emperor of Melody. The Life and Music of Ali Akbar Khan.

H.S.

\section{Comment êtes-vous devenu musicien? Appartenez-vous à une longue lignée de musiciens?}

Notre lignée musicale remonte à Tan Sen, célèbre musicien attaché à la cour de l'empereur Akbar, mais en réalité je ne viens pas d'une famille musicale au sens propre du terme, mais de propriétaires terriens. Mais mon grand-père, Sadhu Khan, était un grand mélomane. Il jouait du sitār. Ma famille vivait au Bengale oriental, aujourd'hui le Bangla Desh. Il y avait là une activité musicale considérable. Chaque année, on y organisait un grand festival de musique d'une durée de plusieurs semaines, auquel participaient les musiciens les plus connus de l'Inde.

Dès sa tendre enfance, mon père aimait la musique. Quand ses parents l'expédiaient à l'école, il se rendait secrètement au temple pour écouter les chants religieux. Il fut puni à chaque fois, mais c'était plus fort que lui. Puis, quand il était encore très jeune, il fit une fugue pour aller à Calcutta apprendre la musique. Il lui fallut du temps pour trouver un maître. Finalement, il fut admis par Nula Gopal, un célèbre chanteur de dhrupad ${ }^{1}$ de l'époque, qui lui conseilla de ne faire que des gammes et des exercices pendant neuf ans. Il lui serait ensuite facile d'apprendre tous les răga. C'est ce que fit mon père.

Juste avant la fin de ces neuf ans, mon père fut découvert par un parent. La famille lui demanda de revenir, ce qu'il refusa de faire. Pour finir, il fut d'accord de leur rendre visite. A son arrivée, il découvrit qu'ils avaient arrangé un mariage

Pour ne pas entraver la lecture de cet entretien, nous avons fait figurer un glossaire des termes vernaculaires en annexe [ndlr]. 
pour lui. Ils espéraient sans doute que son épouse réussirait à le retenir à Tripura. Mais la nuit de noces, il s'enfuit et retourna à Calcutta où il apprit que son guru avait succombé au choléra. Affligé, il décida de ne jamais reprendre l'apprentissage du chant.

C'est ainsi qu'il se tourna vers la musique instrumentale. Il pratiqua plusieurs instruments auprès de divers maîtres. Il apprenait vite. Après quelques mois, tel maître lui disait: Va chez quelqu'un d'autre, je t'ai appris tout ce que je savais. Finalement, mon père arriva à la cour de Rampur dont le musicien attitré était le célèbre Mohamed Wazir Khansaheb. Pendant des semaines, mon père tenta de se faire admettre à la maison du grand musicien, mais en vain. Pour finir, il se jeta devant l'attelage du maharaja. Celui-ci descendit de sa voiture et le fit appeler. Quand il apprit son histoire, il demanda à Wazir Khansaheb de l'écouter. Celuici l'admit comme disciple et, après quelque temps, Allauddin Khansaheb était devenu un de ses disciples favoris; il le considérait comme un fils.

A l'époque, Rampur était une ville très musicale. La plupart de ses habitants étaient musiciens ou parents de musiciens. Mon père avait l'habitude d'inviter des musiciens à boire le thé dans sa chambre; il écoutait attentivement leur conversation musicales et leurs interprétations d'anciennes compositions. Toute la nuit, il étudiait; il attachait ses longs cheveux au plafond pour se réveiller si jamais il s'endormait. Il était très déterminé.

Après de nombreuses années d'étude, Mohamed Wazir Khansaheb envoya mon père en tournée, afin qu'il pût acquérir une expérience de la scène. Mon père rencontra un ami du maharaja de Maihar, qui recherchait quelqu'un pour jouer et enseigner la musique à la cour. En réalité, cela ne disait rien à mon père qui désirait poursuivre ses études. Mais comme le maharaja de Maihar avait obtenu la permission de Wazir Khansaheb, il ne put refuser.

C'est à cette époque que je suis né. Nous, c'est-à-dire ma mère, deux sœurs et moi-même, quittâmes Tripura, au Bengale oriental, pour nous installer à Maihar. Je n'avais que quelques mois. Maihar est un endroit très calme dans l'Uttar Pradesh, connu pour deux raisons seulement: mon père, et le temple de la déesse Sharada. C'est là que j'ai grandi.

J'ai subi un enseignement sévère et discipliné. Depuis l'âge de trois ans, j'apprenais de mon père les fondements de la musique indienne, comme un enfant apprend à parler. Quand j'ai eu neuf ans, mon père décida que je devais me concentrer sur le jeu du sarod. Je m'exerçais dix-huit heures par jour. Une seule fois, je pus rompre cette routine pour quelques semaines, car mon père était en tournée en Europe. Je n'ai pas touché à mon sarod et me suis beaucoup amusé. Quand mon père l'apprit, il interrompit sa tournée pour retourner en Inde et me rappeler à l'ordre. Puis tout recommença: il fallait s'exercer, s'exercer et encore s'exercer. Pendant que je jouais dans ma chambrette, mon père se tenait dans le jardin ou dans la cour pour m'écouter. Il m'avait interdit de m'arrêter de jouer. Un jour, je découvris une astuce: en frappant la corde fortement avec le plectre, je parvenais à la rompre; pendant que je la remplaçais, je pouvais me reposer un peu. Mais il fallut peu de temps à mon père pour comprendre mon jeu; il tendit simplement mon sarod d'une corde plus épaisse. 


\section{Avez-vous le sentiment d'avoir manqué une partie de votre jeunesse ?}

Peut-être. Mais rappelez-vous que là où nous habitions, il n'y avait pas d'autres distractions. La seule chose à faire, à part la musique, c'était des promenades. Je ne connaissais rien d'autre. La grenouille qui n'a jamais quitté son étang ne se languit pas de l'océan. Et peu importe, je peux encore me rattraper!

\section{Comment votre carrière de musicien professionnel a-t-elle débuté?}

Tout d'abord, je jouais beaucoup en concert avec mon père, notamment lors de conférences musicales en Inde. Mon premier concert en solo fut en 1936, à Allahabad. Par la suite, la All India Radio à Lucknow m'engagea comme musicien et directeur musical. Ils m'avaient promis un bon salaire, mais mon père leur dit: Non, donnez-lui la moitié, sinon il sera trop imbu de lui-même. Ainsi, pour arrondir les fins de mois ou manger bien, nous faisions des concerts chez des privés. On s'y rendait à bicyclette, dans la nuit. C'est moi qui pédalait, avec mon sarod comme un poulet mort dans un sac; le joueur de tablā s'agrippait à l'arrière, tandis que le joueur de tambura perchait sur la barre en tenant son instrument dans une main et la torche dans l'autre.

Vers l'âge de vingt ans, je fus engagé à la cour du Raja de Jodhpur. Je devais jouer huit ou neuf heures par jour pour lui, puis enseigner et gérer la station de radio. Quand le maharaja avait des insomnies, il me faisait appeler au milieu de la nuit pour jouer. Après quelque temps, il acheta un magnétophone et m'enregistra. Ainsi je pus dormir. Car j'avais beaucoup de travail.

Mais j'avais aussi la belle vie. Je disposais d'une voiture, de domestiques et de tous les agréments d'une grande cour. J'occupais une grande maison près du palais. J'avais aussi une motocyclette que j'appréciais énormément. Mais lors d'une de ses visites, mon père me demanda une allumette, car il voulait brûler la motocyclette qu'il croyait trop dangereuse. Je lui promis de l'échanger contre une voiture; il fut d'accord.

Pendant mon séjour à Jodhpur, nous organisâmes un grand festival de musique. J'avais invité tout le monde, de toute l'Inde. Dans les temps anciens, seule la famille royale pouvait écouter la musique classique. Mais notre festival se déroula dans une salle de cinéma. Il dura trois nuits. De nombreux vieux musiciens célèbres y participèrent: Hafiz Ali Khansaheb ${ }^{2}$, mon père, et d'autres encore. Puis, après trois jours, le roi demanda aux musiciens de s'attarder un peu, leur proposant de continuer au palais. Cela dura encore une semaine.

Faiyaz Khan ${ }^{3}$, empêché, ne put participer au festival. Nous l'invitâmes plus tard, si bien qu'il chanta au palais pour un petit nombre d'invités et la famille

Hafiz Ali Khan (1877-1972) était un joueur de sarod renommé du début de ce siècle, contemporain de Alauddin Khan et père de l'actuel virtuose Amjad Ali Khan.

3 Faiyaz Khan (1880-1950), un des plus grands chanteurs de ce siècle, était le maître de la gharänā d'Agra. 


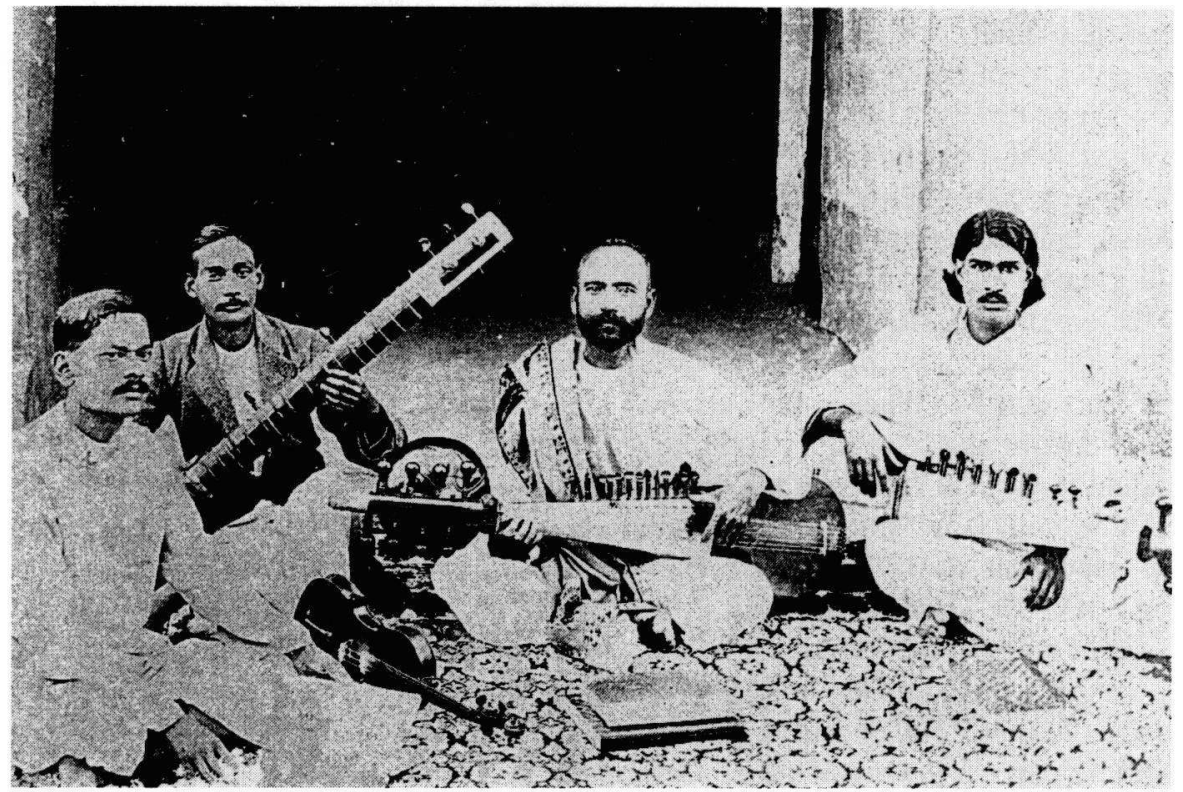

Fig. 1: Allauddin Khan, au centre, avec quelques-uns de ses premiers élèves: Timir Bhattacharya au sarod et un musicien-homéopathe au sitär. Photo: Ali Akbar College of Music, San Rafael, vers 1925.

royale. Après cela, le roi me demanda de jouer du sarod. Je lui répondis: Non, c'est un grand artiste, je ne peux pas jouer après lui ${ }^{4}$. Mais Faiyaz Khan en personne me demanda de jouer, il m'aimait bien. Il me suggéra d'accompagner son chant au sarod, ce que je fis pour lui. Ce fut un concert de trois ou quatre heures. Faiyaz Khan interpréta quantité de rāga: dans les styles dhrupad, khayāl, tarānā, même thumrī. Pour finir, c'était une sorte de duo, nous étions inspirés. Le maharaja en personne nous enregistra, mais la bande magnétique fut perdue.

Pendant tout ce temps, j'étais encore guidé par mon père. Lorsque je faisais une erreur de jeu à la radio, il me téléphonait immédiatement; et à chaque fois que nous nous rencontrions pendant une conférence musicale, il m'enseignait; il suffisait qu'il ouvre le coffre de son sarod pour que je sache que c'était l'heure de la leçon. Cela dura cinquante ans.

\section{Avez-vous réalisé des enregistrements à cette époque?}

Le plus souvent pour la radio. Beaucoup plus tôt, j'avais fait un enregistrement avec mon père pour la Gramophone Company, mais il n'a jamais paru. J'ai commencé à enregistrer à Lucknow pour His Master's Voice, mais je ne me souviens

4 La règle veut en Inde que l'artiste le plus âgé ou le plus considéré joue en dernier. 
pas de l'année. A l'époque, je réalisais des enregistrements d'une durée de trois minutes, comme Darbāri Kanada, Shi rāg, Pilū, Bhairavī.

En ce temps-là, les gens de Calcutta sillonnaient le pays pour enregistrer les artistes. Puis, ils établirent le studio et s'arrangèrent pour que les artistes fassent leurs enregistrements à Calcutta ou à Bombay. Mais auparavant, ils avaient dû louer une maison et prendre contact avec chaque artiste. J'étais le plus jeune. Je jouais avec des musiciens de la All India Radio, le plus souvent avec Wazir Hussein, le dépositaire de la gharāna de Lucknow. J'apparaissais presque chaque semaine à la radio. Parfois, lorsqu'un artiste célèbre nous faisait faux bond, je devais jouer à sa place, même quand j'étais encore très jeune.

Pendant que j'habitais Jodhpur, la plupart de mes enregistrements se faisaient pour la All India Radio où l'on avait créé un système d'engagements en chaîne couvrant toute l'Inde subdivisée en régions: quand j'étais à Jodhpur, je dépendais de la station de Baroda; quand j'étais à Maihar, de la station de Lucknow. Même chose pour Calcutta et Bombay. Si une autre station de radio voulait que je joue pour elle, elle devait en faire la demande à la Ail India Radio.

A cette époque, je jouais plus que d'autres artistes pour la radio; je donnais aussi de nombreux concerts. Tous les deux mois, je me rendais à Baroda, puis à Delhi; parfois il y avait des programmes spéciaux. Je me produisais aussi à Bombay et, bien sûr, à Calcutta. La plupart de mes concerts étaient pour les stations de Lucknow, Calcutta, Bombay et Baroda, plus rarement pour celles de Poona, Madras et Allahabad. Il n'existait alors que peu de stations.

\section{Vous avez aussi travaillé comme directeur musical pour l'industrie du film.}

Après la mort du maharaja dans un accident d'avion, au lendemain de l'Indépendance (1947), je décidai de déménager à Bombay pour travailler pour le cinéma. En fait, un de mes élèves me l'avait suggéré quand j'habitais encore Lucknow. Je voulais atteindre un nouveau public qui n'avait encore jamais écouté de la musique classique. On pouvait aussi gagner beaucoup d'argent en composant des chansons à succès. A l'époque, la musique classique se portait mal à Bombay; il n'y avait que deux groupes d'auditeurs: les milieux du cinéma et les chanteurs. Personne n'avait jamais entendu un sarod. Je voulais remédier à tout cela.

Je n'ai écrit la musique que pour quelques films. Je me souviens que la chanson principale de "Aandhiya» fut interprétée par Lata Mangeshkar ${ }^{5}$. A l'époque, elle avait déjà une expérience telle que si un directeur musical lui téléphonait pour lui demander d'enregistrer une chanson qui commençait de telle manière, elle pouvait immédiatement chanter le deuxième et le troisième vers. Mais pour la chanson en question, nécessitant quatre disques 78 tours, elle passa une semaine en studio pour répéter. Quand la chanson fut enregistrée, elle

Lata Mangeshkar (né en 1929) est probablement l'interprète la plus réputée en Inde du répertoire de la musique légère, et notamment des chansons de films. 
donna tout son argent aux musiciens, tellement elle était contente. Plus tard, la chanson fut récompensée par un prix, mais elle ne devint jamais un succès. De cette manière, je me fis respecter par les musiciens et les critiques.

Mon père, qui n'appréciait pas du tout mon travail pour l'industrie du cinéma, m'écrivit que je n'étais plus son fils. Puis quelques amis l'emmenèrent voir un film d'après une histoire de Tagore, Hungry Stones. Mon père ne savait pas que j'en avais composé la musique. A peine assis, il voulut déjà s'en aller, mais la musique commença. Peu à peu, il se détendit et commença à y prendre plaisir. Quand il apprit à la fin que j'étais le compositeur, il m'écrivit une lettre me donnant la permission de continuer. Mais en réalité, c'est à cette époque que j'ai abandonné l'industrie du cinéma.

Je n'aimais pas ce milieu: les directeurs, les producteurs n'ont aucun sens de la musique. Leur façon d'interférer dans les questions musicales me répugne. Ils versent toujours plus dans le mauvais goût.

\section{Comment votre carrière classique s'est-elle développée à cette époque ?}

J'étais très occupé. Avec Raviji (Ravi Shankar ${ }^{6}$ ), je fus le premier à donner de nombreux concerts en peu de temps et dans toute l'Inde. Parfois, j'allais en deux jours en voiture de Calcutta à Bombay; parfois, je prenais l'avion. A Calcutta, pendant la saison, je donnais parfois trois concerts en un jour: un cercle de musiciens en début de soirée, puis un duo avec Raviji, suivi d'une matinée lors d'une conférence.

J'ai donné beaucoup de concerts avec Ravi Shankar. Nous étions les premiers à jouer en duo, pratique que nous avions développée en jouant avec mon père. Au début, celui-ci nous présentait lors de ses concerts. Puis nous nous sommes mis à jouer seuls. C'était devenu très populaire en Inde.

En Inde, j'ai aussi joué en duo avec Vilayat Khan ${ }^{7}$ que je connais depuis sa jeunesse, quand il apprenait le sitār et ne se produisait pas encore sur scène. Lui aussi est devenu célèbre. Je l'ai invité pour la première fois lorsqu'il était musicien à la cour de Jodhpur. Il resta pendant quelques semaines; j'avais demandé une permission à son altesse, et nous jouâmes presque tous les jours. On nous enregistra: il y avait des heures et des heures d'enregistrements. On avait l'habitude de s'exercer chez moi, car je l'hébergeais. Un jour les bandes magnétiques disparurent. Quand le roi mourut, je les ai demandées à la reine et à d'autres personnes du palais. Finalement on les retrouva, mais tout avait été effacé par un nouvel enregistrement de musique de film.

J'ai aussi joué avec Nikhil Banerjee ${ }^{8}$ qui m'a accompagné pendant quinze ans. Il a vite progressé. Après cinq ou six ans, j'ai commencé à le faire participer à mon

6 Beau-frère d'Ali Akbar Khan (il a épousé sa sœur Annapurna), Ravi Shankar est l'un des plus anciens disciples d'Ustad Allauddin Khan.

7-8 Vilayat Khan (né en 1924) et Nikhil Banerjee (1931-1986) sont, avec Ravi Shankar, les deux sitaristes les plus réputés de leur génération. 
programme et, par ce biais, au festival de musique; nous y avons joué en duo. Puis, dans le Sud, il arrivait que je joue avec un joueur de vinna, un violoniste et un joueur de mridangam - des gens âgés dont j'ai oublié les noms. En Amérique, j'ai joué en duo avec Ravi Shankar, notamment lors de deux concerts de bienfaisance dans des collèges californiens, puis sur la côte est: le fameux concert pour le Bangladesh, et aussi un concert en hommage à mon père après son décès en 1972 .

Maintenant, je ne joue plus en duo, j'aime moins ça de toutes façons. C'est devenu une affaire commerciale: moins de musique, plus de show. Je n'ai jamais aimé le show. Cela nous fait perdre le sens de la vraie musique, ce qui m'a toujours dérangé. Il faut choisir des rāga légers. Si on joue des rāga complexes, le partenaire ne répond pas toujours comme il faut. Mais avec Panditji (Ravi Shankar), cela allait bien. Dans notre jeunesse, nous avons joué quelques jolis duos.

\section{Lors d'un entretien de trois heures que Buddadev Dasgupta eut avec vous pour la All India Radio, celui-ci semblait dire que dans le domaine du sarod, il y a un avant et un après Ali Akbar. En quoi votre style est-il distinctif?}

En fait, ce n'est pas mon style mais celui de mon père, c'est l'apprentissage qu'il fit lui-même et qu'il me transmit par la suite. Ayant appris le jeu de nombreux instruments chez autant de maîtres, il tenta d'appliquer tout cela dans le jeu du sarod. Bien sûr, il m'a appris beaucoup dans le domaine du chant. Mais il lui arrivait de jouer un morceau au piano ou au violon, puis de me demander de le jouer au sarod.

Il créa aussi un système: ālāp en tant de parties, gat en tant d'autres. En écoutant les anciens maîtres, on constate qu'il n'y avait jadis que peu de diridiri, quelques jhāla, tihā et layakāri, c'était tout. Aussi, chacun était spécialisé: si on voulait écouter de bons tān, jhäla ou àlāp, il fallait aller vers tel ou tel musicien. Mais mon père réussit à combiner tout cela.

Il n'eut pas le temps de parfaire son style; c'est pourquoi il l'essaya sur moi. Je me suis lancé. Je l'ai développé. Maintenant, quelle que soit leur gharāna, les sarodistes suivent mon style: le son, la technique, les rāga les gat. C'est comme en médecine: après avoir essayé ceci ou cela, on découvre le médicament.

\section{Vous avez aussi fourni votre propre contribution à la musique indienne: de nou- veaux rāga comme Chandranandan et Gauri Manjari.}

C'est à Bombay que j'ai enregistré Chandranandan, Gauri Manjari et d'autres rāga pour la première fois. J'ai composé Chandranandan sur-le-champ. M. Joshi, alors responsable de His Master's Voice, avait une vaste connaissance de la musique indienne et pratiquait le chant. Un jour, il me fit savoir qu'il avait envie d'écouter quelque chose d'inédit. J'étais jeune, voyez-vous, je songeais à tous ces rāga qu'il connaissait sans doute, mais comme il s'attendait à ce que je joue quelque chose, j'ai joué. En trois minutes, j'ai composé au gré des notes. Je me disais que rien de grave ne pouvait se passer en trois minutes, et que si c'était vraiment mauvais, on l'effacerait. Mais c'était bon; ils ne l'ont pas supprimé. Ils ont enre- 


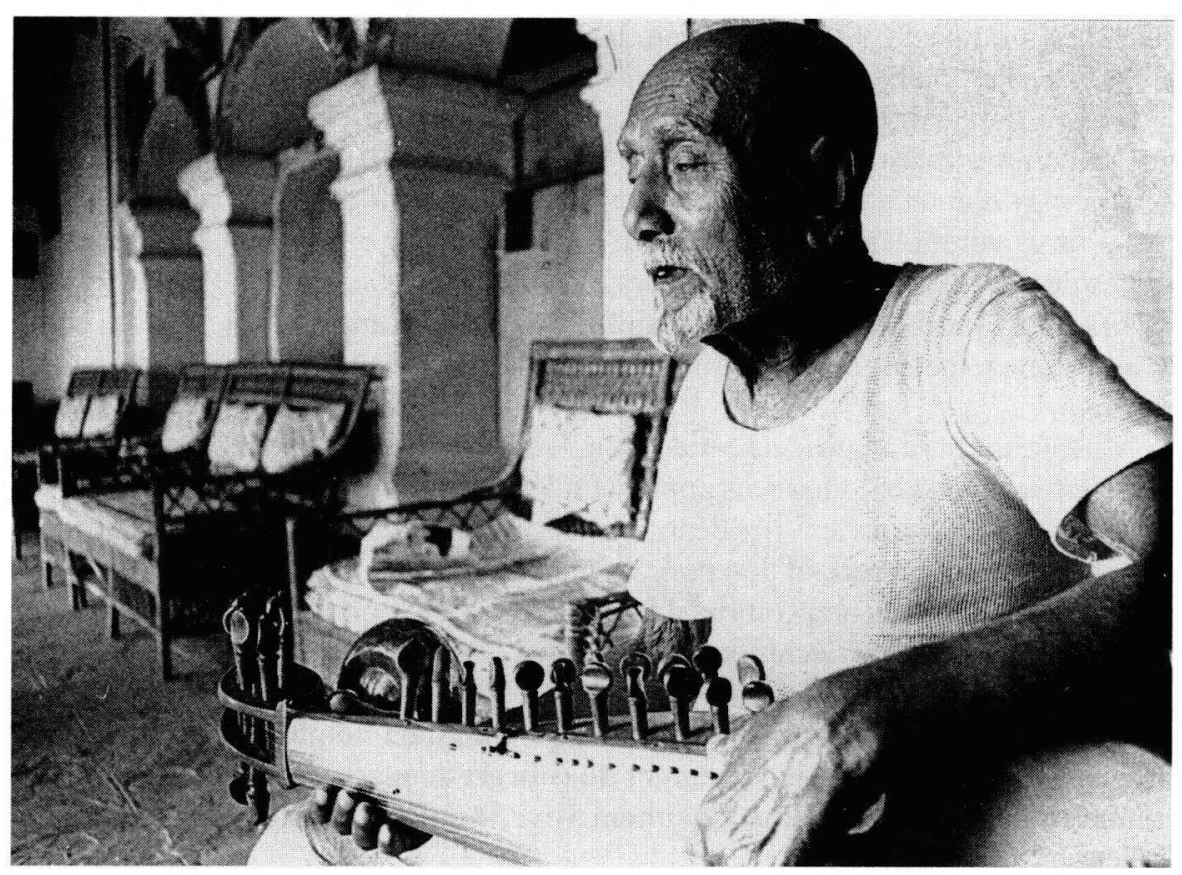

Fig. 2: Allauddin Khan à Maihar.

Photo: Ashish Khan, s.d.

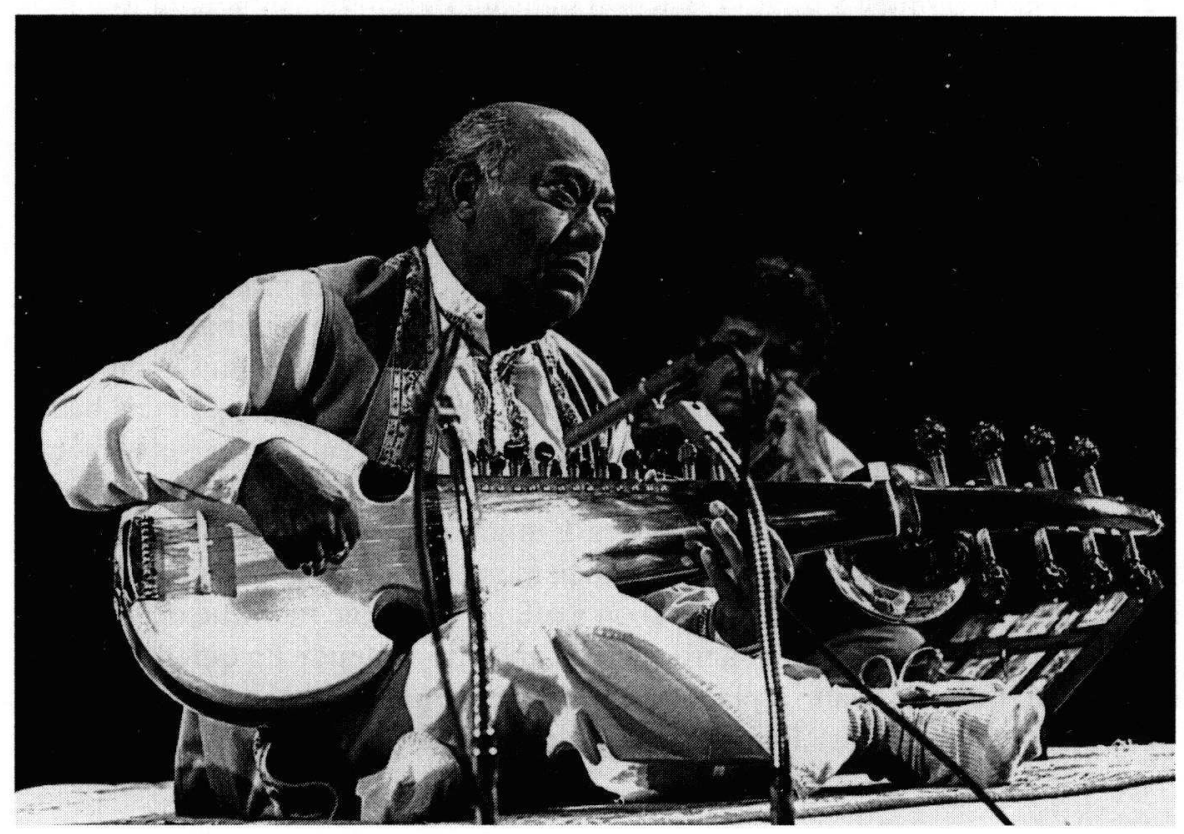

Fig. 4: Ali Akbar Khan en concert à New York.

Photo: Ira Landgarten, 1987. 
gistré le tout. Puis M. Joshi me demanda de donner un titre; je ne savais pas comment l'appeler, mais je lui dis que ce rāga me faisait penser à la lune ${ }^{9}$.

A la sortie du disque quelques mois plus tard, j'avais déjà oublié tout cela. En quelque jours, le disque fut épuisé et il fallut le presser à nouveau. Puis le public de Bombay commença à réclamer Chandranandan. Alors j'ai dû acheter le disque pour écouter et apprendre ce răga. Il m'a fallu cinq ans pour le comprendre. Après douze ans, j'avais trouvé la bonne façon de le jouer.

Quant à Gauri Manjari, je l'ai planifié. Je voulais composer un rāga qui combine les douze notes, mais j'ai dû m'arrêter à onze pour que cela sonne comme un rāga.

Chaque artiste compose de cette façon. Je n'ai rien inventé. Mais après vingt ou cinquante ans, on voit si les gens apprécient les compositions, si elles figurent dans les livres. Je ne me souviens pas de tous les rāga que j'ai composés. Composer, c'est une chose; il faut un certain don pour voir clairement. Mais quand il s'agit d'enseigner ou de rejouer la composition, il faut se mettre à l'apprendre vraiment. C'est à ce moment qu'il faut réfléchir et garder l'esprit critique.

\section{Au cours de votre vie, la musique indienne a connu de nombreuses transforma- tions: le déclin des cours, l'avènement du concert de masse, la radio, les enregis- trements, la musique de film. Comment voyez-vous l'état actuel de la musique indienne?}

Auparavant, lors d'un concert, les premiers rangs étaient occupés par des musiciens et des mélomanes dont la réaction était un baromètre de la qualité de la représentation. S'ils aimaient un jeune musicien, ils montaient sur scène pour l'embrasser; s'ils lui trouvaient des erreurs, ils le huaient; il arrivait même qu'ils frappent l'artiste. C'est ce que faisait mon père. C'était bon pour la musique. Maintenant, des gens riches en complet et sari coûteux sont au premier rang. Les vrais mélomanes doivent se contenter des places du fond de la salle, avec le résultat que les musiciens deviennent insouciants et essayent surtout d'impressionner l'auditoire par un jeu très rapide. Mais ce n'est pas la vraie musique.

Dans ma jeunesse, de nombreux musiciens confirmés venaient à Maihar. J'ai entendu tous les musiciens célèbres dans tous les festivals organisés en Inde. J'assistais à des concerts qui duraient toute la nuit. A l'époque, j'aimais beaucoup cela, mais maintenant je n'y assiste plus, car je ne rencontre plus la même qualité chez la jeune génération. La quantité augmente, mais la qualité diminue. Tout le monde cherche à imiter les autres. L'imitation est une limitation. On peut faire briller différentes choses comme un diamant, mais un vrai diamant est facile à reconnaître. On voit tout de suite si on a affaire à la vraie musique ou non.

Jadis, les musiciens étaient amis. Je n'en ai jamais entendu un qui dise du mal d'un autre. Chacun avait sa place et personne ne pouvait y toucher. Les musiciens étaient autonomes; ils apprenaient, s'exerçaient, se produisaient en public.

9 «Chandranandan» signifie «béatitude (ānanda) de la lune (chandra)». 
Les gens venaient simplement les écouter, non les comparer. Chaque musicien avait sa façon d'interpréter un rāga, sa propre technique et son propre doigté; chacun exprimait ses propres sentiments à sa manière. Aujourd'hui, on constate une tendance à voler tel détail à l'un, tel détail à l'autre. Jadis, le musicien savait conserver son style.

De nos jours, l'approche des rāga et du style manque de profondeur. Les joueurs veulent surtout faire état de leur virtuosité; ils veulent être magiciens, non musiciens. C'est un manque de concentration et d'entraînement. Chacun veut rapidement devenir célèbre. Il y a beaucoup de compétition. Tous les jeunes sitaristes veulent devenir comme Vilayat Khan, Ravi Shankar ou Nikhil Banerjee en l'espace d'un mois. Cela s'applique aussi aux chanteurs: ils veulent être comme untel, mais en imitant untel, qu'apportent-ils de neuf? Jadis, personne ne ressemblait à personne. Si je veux écouter Vilayat Khan ou Ravi Shankar, pourquoi t'écouter?

\section{Pensez-vous que la musique classique de l'Inde est en danger?}

Non, pas vraiment. Il y aura toujours des musiciens authentiques qui persévèreront, peu importe les difficultés qu'ils rencontrent. La musique classique nourrit l'âme et le corps; elle confère la paix intérieure. La musique, c'est le langage des dieux; elle vous rapproche d'eux.

Les problèmes que la musique indienne connait actuellement semblent surtout être liés à l'enseignement. Les grands musiciens sont continuellement en tournée, ils n'ont pas de temps pour leurs élèves. Vous, au contraire, semblez attacher beaucoup d'importance à la transmission de votre savoir.

Je ne pense pas qu'il faille dépenser toute son énergie en concert; cela porte atteinte à la qualité; il y a le danger du jeu mécanique. Mon objectif principal est l'enseignement; c'est mon devoir. Je ne puis garder pour moi ce que je possède: il me faut le transmettre. Jusqu'à l'époque de mon père, les maîtres avaient l'habitude de n'enseigner qu'à leurs fils, même s'ils n'étaient pas doués. Ils n'ont jamais songé s'entourer de disciples. Les fils avaient l'habitude d'entendre leur père dès la petite enfance; c'est ainsi qu'ils apprenaient. Mais il n'existait aucune méthode d'enseignement. Nombreux furent les maîtres anciens qui ne transmettaient jamais le titre d'un rāga, les échelles ascendantes et descendantes, les $v \bar{a} d i$ et les samvādi. Ils enseignaient la musique par la pratique; c'était comme si on faisait le même chemin chaque jour; ce chemin mène quelque part, sans doute, mais on n'y apprend pas grand-chose. Quant à mon père, il faisait des recherches sur tout; il passa quarante ans à apprendre, et quarante ans à enseigner et à jouer en public.

Après la mort du roi de Jodhpur, en 1956, j'ai fondé à Calcutta le Ali Akbar College of Music. Parfois, ma sœur Annapurna y enseigne encore. Puis, dans les années soixante, un grand nombre d'élèves occidentaux sont venus en Inde. J'ai découvert qu'il était plus facile qu'un guru prenne l'avion, plutôt que des centai- 
nes d'élèves. C'est ainsi que le Ali Akbar College of Music de Californie ouvrit ses portes en 1967. J'y enseigne trois ou quatre jours par semaine pendant une bonne partie de l'année.

La plupart des élèves sont des Occidentaux; certains d'entre eux apprennent maintenant depuis dix, voire quinze ans. Certains m'assistent dans l'enseignement; je n'ai plus besoin d'assistants venant de l'Inde. J'enseigne comment jouer sur scène à ceux de mes élèves que je crois capables. Je l'ai même fait pour Nikhil Banerjee. Ici, les élèves ont la possibilité de jouer en public. Mais il faut qu'ils aient atteint un certain niveau; il faut qu'ils soient vraiment de bons musiciens, ce qui est plus difficile dans son pays d'origine. Les gens vous acceptent si vous avez gagné une certaine réputation et expérience à l'étranger. Un jour, il y aura de bons sarodistes américains.

\section{Quelle est le mode d'apprentissage de vos élèves? Prennent-ils des notes?}

Je n'aime pas que les élèves prennent des notes; il ne faut pas qu'ils écrivent pendant qu'ils apprennent. Ils peuvent écrire, mais à la fin, avant de rentrer chez eux. Il vaut mieux enregistrer, car en écrivant, on passe à côté de la partie cruciale de l'enseignement, l'apprentissage en présence du maître. Dans d'autres écoles, on enseigne sur la base de livres; c'est une approche tout à fait différente de celle qu'on appelle guru vidya mukhya, l'apprentissage de bouche à oreille. C'est comme la différence qu'il y a entre la nourriture que la mère prépare pour les enfants et un plat congelé. Sous ce rapport, la cassette convient mieux. En prenant des notes, on fais travailler les yeux. Quand on n'écrit pas, on écoute simplement la musique, on observe le maître et c'est ainsi qu'on apprend: voilà la vraie musique. Et on exerce, non pas la vue, mais l'ouïe. En se limitant à la vue, on passe à côté de l'émotion; c'est comme si vous regardez la photo d'un être cher, alors qu'il est à côté de vous .

Je n'ai jamais pris de notes moi-même: pas de stylo, pas de bloc-notes. J'ai appris sur le tas, excepté quelques séances pour lesquelles mon père se servit de ses ouvrages anciens. Après nous avoir enseigné des centaines de dhrupad et de dhamār, il nous autorisa, ma sœur et moi, à commencer à écrire. Il nous procura des carnets, des crayons, des stylos. Il prenait un de ses livres et se mit à chanter, une portée à la fois. Ma sœur s'acharnait à transcrire. Entre-temps, je dessinait un âne, un oiseau. Mais il fallait chanter. Je chantais comme un merle, tout en mémorisant la page et la couleur du livre. Lorsque mon père se rendait chez le roi ou au marché, je copiais la pièce sur son livre, je savais où il le gardait. Mes transcriptions étaient toujours bonnes, puisque je les copiais sur les siennes. Mais il arrivait que mon père n'aille pas au marché, peut-être parce qu'il pleuvait, ou pour une autre raison. Ainsi, un jour, il me découvrit. Ma sœur avait éclaté de rire, car j'étais en train de dessiner un âne. A la question de mon père sur la raison de son hilarité, elle répondit que c'était à cause de moi; mais mon père ne voyait rien d'amusant. Il était assis sur une petite plate-forme, tandis que ma sœur et moi étions assis par terre. Soudain, il m'appela pour lui montrer mon carnet. Quand il le vit, il sourit, mais il était aussi très fâché. 


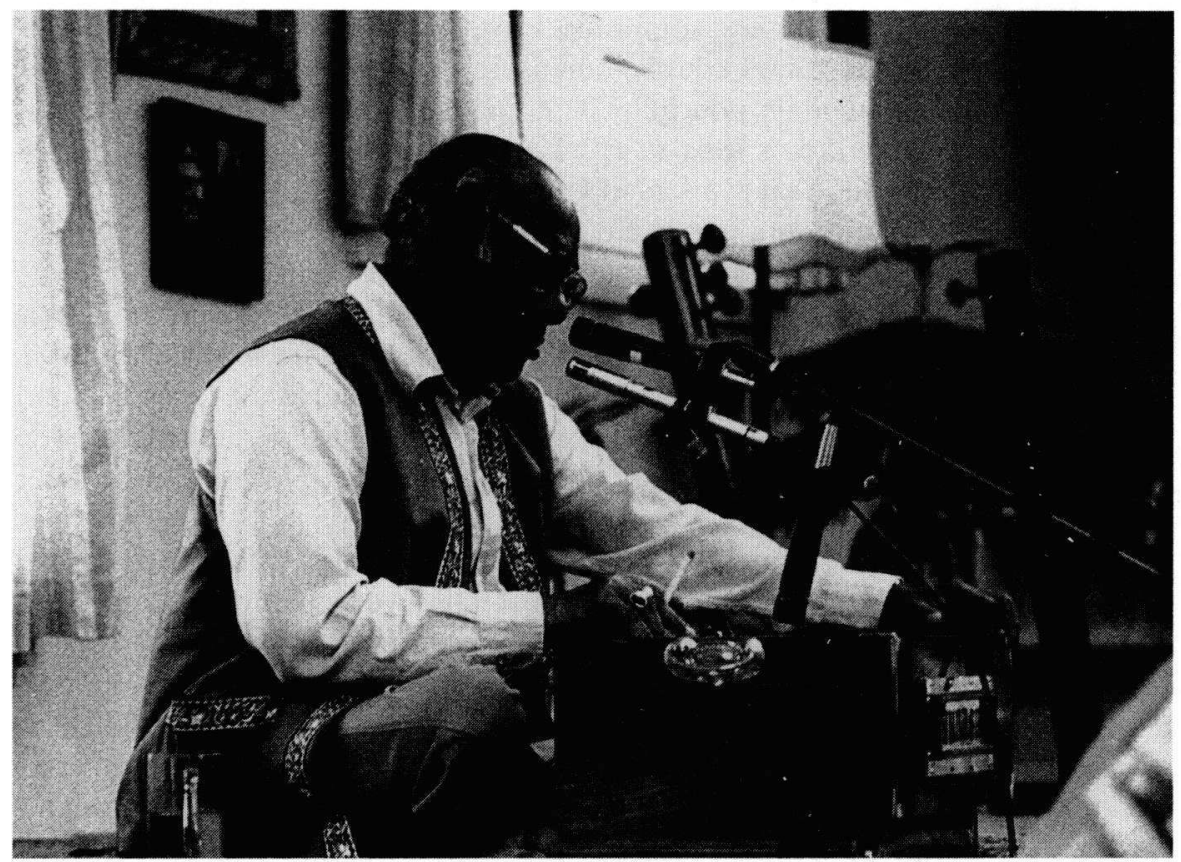

Fig. 3: Ali Akbar Khan enseignant à l'aide de l'harmonium au Ali Akbar College of Music, San Rafael, Californie. Photo: Marjolein van Velzen, 1987.

Depuis vingt-cinq ans, vous vivez en Occident. Pouvez-vous dire quelque chose à propos de vos expériences ici?

En 1955, mon ami Yehudi Menuhin m'invita pour des concerts et des enregistrements. Je passai aussi à la télévision. A l'époque, ces auditeurs ne connaissaient pas encore ma musique, et je les ignorais tout autant. Le chef d'orchestre Stokowski n'assista pas à mon premier concert au Museum of Modern Art parce qu'il croyait que toute la musique indienne était comme la musique populaire qu'il avait entendu dans les enregistrements d'Alain Daniélou. Mais il vint par la suite à mon concert au Rockefeller Center. Après le concert, il s'excusa pour son erreur.

Plus tard, dans les années soixante, des choses bizarres se passèrent. Pendant cette période, j'ai délibérément gardé mes distances. Je n'aimais pas l'intérêt superficiel manifesté à l'époque. Je préfère des auditeurs sérieux. Je ne voulais pas changer de style, par exemple en jouant des pièces plus brèves, car cela portait atteinte à la musique.

Mais quand je suis venu enseigner ici, j'étais bien disposé à l'égard des filles et des garçons désireux d'apprendre la musique: ils étaient si doués, si gentils, si polis. Je ne voyais rien de mauvais. J'en avais entendu parler, mais je ne voyais 
rien. Peut-être dans les classes nombreuses avec cent cinquante élèves, un ou deux avait des cheveux longs et des vêtements sales. De l'Inde, je connaissais les $s \bar{a} d h u$, et je me demandais pourquoi ils copiaient les sādhu de Bénarès. Ils avaient l'air de $s \bar{d} h u$, mais sans se comporter comme eux. La saleté ne me dérangeait pas non plus, j'en avais aussi vu en Inde.

Maintenant, j'ai un grand nombre d'élèves occidentaux à travers le monde. Chaque année, je viens en Europe pour enseigner dans la filiale de mon école que Ken Zuckerman a fondée en Suisse, et pour donner des concerts. Je passe aussi deux ou trois mois en Inde. J'ignore combien de temps je ferai encore cela, c'est si compliqué. Cette fois-ci, j'ai été coincé par la guerre du Golfe. Quand mon père et ma mère étaient encore en vie, il fallait retourner en Inde chaque année. Maintenant, je dois m'y rendre pour mon auditoire. Je continue à jouer en concert partout.

L'Inde m'a demandé de rentrer. Le Premier Ministre m'a offert toutes sortes de facilités pour ouvrir une école à Delhi. Le gouvernement de Bengale, à Calcutta, veut aussi quelque chose. D'une manière ou d'une autre, je me débrouille. Mais j'ai l'intention de rester ici. J'y vais en visite. J'ai mis un terme à mes activités au Ali Akbar College à Calcutta qui continuera sous le nom de mon père, en sa mémoire. Le Bengale m'a offert un terrain pour construire une école. Un jour, j'irai. Mes fils y sont; tous mes disciples, ceux qui sont bons, peuvent s'y rendre. Ma sœur peut y aller. Je veux aussi qu'il y ait une bonne bibliothèque et de bonnes archives. Mais je suis content de rester ici [en Californie]. 


\section{Glossaire}

La translitération et la définition de ces termes sont basées sur celles données dans l'ouvrage de Patrick Moutal: HindusthānI Rāga Sangīta. Une étude de quelques mécanismes de base. Ouvrage publié avec le concours du Centre d'études de musique orientale (tirage limité). Paris. 1987. Les voyelles figurant entre parenthèses sont écrites en hindi, mais ne se prononcent pas [ndlr].

Alāp(a) Prélude instrumental d'un rāga, interprété sans percussions.

Dhamār(a) Forme de composition dans le style dhrupad, interprété dans le cycle rythmique du même nom, à 14 temps.

Dhrupad(a) Texte poétique mesuré; genre vocal et instrumental sérieux et orthodoxe.

Diridiri Onomatopée désignant, dans le jeu des instruments à cordes pincées, un double mouvement d'aller et retour rapide du plectre ou de l'onglet sur une corde.

Gat(a) Composition instrumentale rythmée dans un Faga.

Gharāna École traditionnelle de la musique vocale ou instrumentale; style de cette école.

Guru vidya mukhya Transmission orale du savoir d'un maître.

Jhālā Troisième et dernière partie de l'ālāp, caractérisée par son tempo rapide et l'importance de ses formules rythmiques; le jhâlā est également joué avec l'accompagnement du tablā après l'élaboration de la composition rapide.

Khayāl(a) Litt. «imagination» en arabe. Genre classique de la musique vocale, et par extension instrumentale, caractérisé par de longs développements improvisés.

Komal(a) Bémol.

Layakārī Jeu sur les tempos, forme de polyrythmie.

Mridangam Tambour en forme de tonneau utilisé dans la musique carnatique du Sud de l'Inde.

$R \bar{a} g$ (a) Litt. «coloration de l'esprit» en sanscrit. Etre musical ayant une forme et un esprit définis par la tradition. Ses éléments constitutifs principaux sont: échelle ascendante et descendante, hiérarchisation des notes, notes de passage, phrases caractéristiques, prédominance d'octaves ou de tétracordes et mouvements mélodiques.

Sādhu Ascète hindou menant une vie érémitique.

Samvādī Litt. «co-parlante», seconde note accentuée d'un rāga, distante d'une quarte ou d'une quinte de la vādi.

Sarod Instrument de l'Inde du Nord, à cordes pincées à l'aide d'un plectre. Sa caisse de résonance est recouverte d'une peau, et son manche d'une touche métallique.

Sitār Instrument de l'Inde du Nord à cordes pincées à l'aide d'un onglet. Sa caisse de résonance est en calebasse et son long manche est pourvu de frettes métalliques.

$\operatorname{Suddh}(a)$ «Pur», se dit d'une note non altérée.

Tan(a) Trait mélodique.

Tarānā Composition vocale très rapide utilisant des onomatopées.

Thum(a)rī Genre vocal semi-classique, généralement interprété dans certains rāga dits légers.

Tihā̄ Formule rythmique répétée trois fois, dont la dernière se termine en un point déterminé du cycle rythmique.

$V \bar{a} d \bar{\imath}$ Litt. «parlante», la note la plus importante d'un rāga, distante d'une quarte ou d'une quinte de la vamvādì.

Viña Instrument à cordes de l'Inde, soit luth, soit cithare sur bâton, dont on rencontre plusieurs variantes dans le Nord et le Sud du pays. 


\section{Discographie}

Editions en microsillons (LP), en cassettes (MC) et en disques compacts (CD), classés chronologiquement selon l'année d'enregistrement, lorsqu'elle est connue.

Rāga Sindhi Bhairavi

Tablā: Chatur Lal

In: «Anthologie de la Musique de l'Inde», vol. 1 , face $B$, plage 4

Ducretet Thomson 320c096 (1955).

Réédition Disques GREM, G 1508 (1985)

«Music of India»

Rāga Sindhi Bhairavi

Rāga Pilū

Avec une introduction parlée

par Yehudi Menuhin.

Tablā: Chatur Lal

Edition originale Angel.

Réédition Gramco India,

LP ALPC 2 (1956; enreg. 1955)

Rāga Todi

Rāga Lajwanti

Gramco India, LP EALP 1255 (1961)

Rāga Chandranandan

Rāga Bhairavi

Tablā: Shashi Bellari

Gramco India, LP EALP 1268 (1962)

Rāga Yaman Kalyan

Tablā: Shashi Bellari

In: «Classical Indian Music», vol. 2, face A

Odeon, coffret de 3 LP PMAE 501/2/3 (1962)

Rāga Ramdasi Malhar

Rāga Pilu

Tablā: Nikhil Ghosh, Shashi Bellari

Gramco India, LP EALP 1274 (1963)

Rāga Nat Bhairav

Rāga Asavari

Rāga Jaijaiwanti

Rāga Bilaskhani Todi

Rāga Malkauns

Rāga Kaushi Kanada

Rāga Darbari Kanada

Regal/Gramco India, LP D/ELRZ 8 (1969);

(Réédition de disques 45t (1961-1964)

Rāga Pahadi Jhinjhoti

Rāga Mishra Shivaranjani

Tablā: Chatur Lal

Gramco India, LP EALP 1281 (1964)
«North Indian Master»

Rāga Mishra Mand

Rāga Basant Mukhari

Rāga Darbari Kanada (ālāp)

Tablā: Shankar Ghosh

World Pacific, LP WPS 21433 (1965)

Rāga Prabhakali (dhamār)

Rāga Kaushi Bhairavi

Tablā: Kishan Maharaj

Gramco India, LP EALP 1286 (1965)

Rāga Mian ki Todi

Rāga Zilla Kafi

Tablā: Shankar Ghosh

Gramco India,

LP EASD 1290 (1965)

Rāga Multani

Rāga Hindol Hem

Tablā: Shashi Bellari

Gramco India,

LP EALP 1301 (1965)

Rāga Durga

Rāga Khammaj (thumrī)

Tablā: Shankar Ghosh

Gramco India,

LP EALP 1310 (1966)

«Sound of the sarod»

Rāga Kirwani

Rāga Chandranandan

Tablā: Shankar Ghosh

World Pacific, LP WPS 21435 (1966),

MC Ravi Shankar Music Circle RSMC 43

«Master Musician of India »

Rāga Chandranandan

Räga Gauri Manjari

Tablā: Mahapurush Mishra

Connoisseur Society,

LP CS 462 (1966)

«Morning and Evening Rāgas »

Rāga Gujari Todi

Rāga Mishra Mand

Tablā: Mahapurush Mishra

Connoisseur Society,

LP CS 1766 (1966) 
«Predawn to Sunrise Rāgas»

Rāga Bairagi

Rāga Ahir Bhairav

Tablā: Mahapurush Mishra

Connoisseur Society, LP CS 1967 (1967)

Rāga Basant Mukhari

Tablā: Shankar Ghosh

In : «The Anthology of Indian Music», face B

World Pacific, LP WPS 26200 (1967), Ravi

Shankar Music Circle, MC RSMC 23

Rāga Medhavi

Rāga Bairagi

Tablā: Shankar Ghosh

Gramco India, LP EASD 1319 (1967)

Rāga Desh Malhar (ālāp)

Rāga Desh (gat)

Rāga Nat Bhairav

Gramco India, LP EASD 1324 (1967)

«The Forty Minute Rāga»

Rāga Marwa

Tablā: Mahapurush Misra

Connoisseur Society, LP CS 2008 (1968)

«Rāgmāla»

Rāga Jogiya Kalingra

Rāga Bhairavi Bhatiyar

Tablā: Mahapurush Mishra

Connoisseur Society, LP CS 2011 (1969)

«The Eighty Minute Rāga»

Rāga Kanada Prakar

Tablā: Mahapurush Mishra

Connoisseur Society, LP CS 2012 (1969)

«Shree Rāg»

Rāga Shree

Tablā: Shankar Ghosh

Connoisseur Society, LP CS 2015 (1969)

«Rāgas of India»

Rāga Madhabi

Rāga Khammaj

Tablā: Mahapurush Mishra

Connoisseur Society, LP CS 2020 (1970)

«Bangla Desh»

Rāga Bhimpalasi

Rāga Mishra Shivaranjani

Tablā: Shankar Ghosh

Connoisseur Society, LP CS 2042 (1972)

«The Jewels of Maihar"

Rāga Darbari Todi

Rāga Bhupali Todi

Tablā: Mahapurush Mishra

Alam Madina, MC AMMP CS 82-3

(1982; enreg. ca 1970)
Rāga Ahir Bhairav

Rāga Durgeshwari

Tablā: Zakir Hussain

Gramco India, LP EASD 1391 (1973)

Rāga Alamgiri

Rāga Jogiya Kalengra

Tablā: Swapan Chaudhuri

Gramco India, LP EASD 1407 (1974)

«Music for Meditation»

Rāga Bilaskhani Todi (ālāp)

Connoisseur Society, LP CS 2063 (1974)

«Live at the Ali Akbar College of Music»

Rāga Shudh Kalyan (ālāp)

Rāga Durga

Rāga Gorakh Kalyan

Tablā: Jnan Prakash Ghosh

AACM (1977; enreg. 1977)

Rāga Malayalam

Tablā: Swapan Chaudhuri

Gramco India, LP ECSD 2548 (1977)

«Evening Rāgas in San Francisco»

Rāga Pahadi Jhinjhoti

Rāga Durgeshwari

Tablā: Zakir Hussain

Alam Madina, MC AMMP CS 79-1 (1979)

Rāga Suha Todi

Rāga Chhayanat

Tablā: Swapan Chaudhuri

Gramco India, LP ECSD 2587 (1980)

«Live in Berkeley»

Rāga Darbari Kanada

Rāga Madan Manjari

Rāga Marwa

Rāga Paraj Basant

Tablā: Pranesh Khan

Alam Madina, MC AMMP CS 80-2 (1980)

«From the Concert Hall»

Rāga Shuddh Kalyan

Rāga Jhinjhat Manj

Tablā: Kishan Maharaj

Gramco India, LP ECSD 2599 (1980)

«Live in Calcutta, Part I»

Rāga Nat Bhairav

Tablā: Swapan Chaudhuri

Alam Madina, MC AMMP/CS86-4

(1987; enreg. 1981)

«Live in Calcutta, Part II»

Rāga Sindhi Bhairavi

Tablā: Swapan Chaudhuri

Alam Madina, MC AMMP/CS86-5

(1987; enreg. 1981) 
«Live from Delhi»

Rāga Mian ki Malhar

Tablā: Shankar Ghosh

Alam Madina, MC AMMP/CS 86-7

1987; enreg. 1981)

«Soul of the Sarod»

Rāga Lalit, Tilak Kamod

Tablā: Swapan Chaudhuri

Oriental Records, LP BGRP 1041,

CD AAMS CD 128 (1982)

«Half Moon»

Rāga Chayanat

Rāga Malashri

Tablā: Swapan Chaudhuri

Metalanguage, LP ML 122 (1983)

«In Eugene, Oregon»

Rāga Hem Bihag (ālāp, jor)

Rāga Bihag (gat)

Tablā: Swapan Chaudhuri

Alam Madina, MC AMMP/CS86-6

(1987; enreg. 1983)

«The Great Genius of Sarod »

Rāga Lome

Rāga Bihag

Tablā: Swapan Chaudhuri

Chhanda Dhara, LP SP 9083 (1983)

«Ali Akbar Khan Concert »

Rāga Kaushi Kanada

Rāga Chandranandan

Tablā: Swapan Chaudhuri

AACM, MC RTC 83.1 (1983)

«Allauddin Khan Memorial Concert »

Rāga Darbari Kanada

Rāga Kirwani

Tablā: Swapan Chaudhuri

AACM, MC RTC 83.2, partie 1 (1983)

«Allauddin Khan Memorial Concert »

Rāga Mishra Mand

Tablā: Swapan Chaudhuri

AACM, MC RTC 83.2, partie 2 (1983)

«Ali Akbar Khan in Concert», vol. 4

Rāga Mian ki Sarang

Rāga Bhoop Mand

Tablā: Mahapurush Misra

AACM, MC RTC 83.4 (1983)

«Ali Akbar Khan in Concert», vol. 5

Rāga Hindol Hem

AACM, MC RTC 83.4 (1983)
Räga Bageshree Kanada

Tablā: Mahapurush Mishra

Gramco India, LP EASD 1419 (1983)

«The Summer Solstice Concert»

Rāga Nat Bhairav

Tablā: Swapan Chaudhuri

AACM, MC RTC 8 (voir Vidéo) (1983)

Rāga Desh Malhar

Tablā: Swapan Chaudhuri

AACM, MC RTC 9 (1983)

Rāga Jogia Malhar

Tablā: Swapan Chaudhuri

AACM, MC RTC 10 (1983)

Rāga Kirwani

Tablā: Swapan Chaudhuri

Water Lily Acoustics LP ES-01 (1984)

«Artistic Sound of Sarod»

Rāga Basant Mukhari, avec Jogiya

Tablā: Swapan Chaudhuri

Chhanda Dhara, LP 10685 ,

MC SP 10685m, CD SN 3386 (1985)

«In Concert at St. John's»

Rāga Mishra Kirwani

Tablā: Zakir Hussain

Alam Madina, MC AMMP/CS86-6

(1987; enreg. 1985)

«Live in Amsterdam», partie 1

Rāga Pahadi Jhinjhoti

Tablā: Swapan Chaudhuri

MA Cassettes, MC MA 8511 (1985)

«Live in Amsterdam», partie 2

Rāga Jog

Rāga Bhairavi

Tablā: Swapan Chaudhuri

MA Cassettes, MC MA 8512 (1985)

«Rare Artists, Rare Rāgas»

Rāga Darbari Todi

Rāga Mian ki Sarang

Rāga Mishra Gara (seulement sur le CD)

Tablā: Swapan Chaudhuri

Chhanda Dhara, LP SP 11886,

MC SP 51886, CD SNCD 21086 (1986)

«Maihar»

Rāga Yaman Kalyan

Rāga Jog

Tablā: Swapan Chaudhuri

Water Lily Acoustics LP ES-10

(1990; enreg. 1989) 
«New Recording»

Rāga Lankadahan Sarang

Rāga Maligaura

Rāga Tilak Kamod ki Prakar Rāga Tilak Nat

Rāga Tilak Kamod

Rāga Tilak Bihari

Tablā: Swapan Chaudhuri

Gramco India CD PSLP 5161 (1990)

Rāga Puriya Kalyan

Rāga Anheri Bhairavi

Tablā: Swapan Chaudhuri

Gramco India MC

«Signature Series», vol. 1

Rāga Chandranandan

Rāga Gauri Manjari

Rāga Jogiya Kalingra

AMMP 9001

(réédition d'enregistrements

de la Connoisseur Society)

«Signature Series», vol. 2

Rāga Medhavi

Rāga Khammaj

Rāga Bhairavi Bhatiyar

AMMP 9002

(réédition d'enregistrements

de la Connoisseur Society)

«Emperor of Sarod»

Rāga Bageshri Kanada

Tablā: Swapan Chaudhuri

Chhanda Dhara (1991)

\section{Bandes vidéo}

«The Summer Solstice»

Rāga Nat Bhairav

Rāga Suha Sughrai

Rāga Mishra Mand

Rāga Brindabani Sarang

Rāga Zilla Kafi

Tablā: Swapan Chaudhuri

Alam Madina Video V-88-1 (1981)

«Concert at St John's in Berkeley», partie 1

Rāga Manjari Malhar

Rāga Pahadi Jhinjhoti

Tablā: Zakir Hussain

Sound Photosynthesis

SP 9052-87 (1987)

«Concert at St John's in Berkeley», partie 2

Rāga Zilla Kafi

Tablā: Zakir Hussain

Sound Photosynthesis

SP 9053-87 (1987)
«Concert at First Unitarian Church

in San Francisco », partie 1

Rāga Khambavati Kanhra- Khammoji

Tablā: Swapan Chaudhuri

Sound Photosynthesis SP 9034-87 (1987)

«Concert at First Unitarian Church in San

Francisco ", partie 2

Rāga Malkauns

Tablā: Swapan Chaudhuri

Sound Photosynthesis SP 9035-87 (1987)

«Concert at Julia Morgan Theatre in

Berkeley»

Rāga Basant Mukhari

Rāga Bhairavi Bhatiyar

Tablā: Swapan Chaudhuri

Sound Photosynthesis

SP 9091-88 (1988)

\section{Duos avec Ravi Shankar}

Rāga Manj Khammaj

Tablā: Chatur Lal

In: "Anthologie de la Musique Classique de l'Inde», vol. 1, face B,

Disques GREM, LP G 1508 (1955)

«Music of India»

Rāga Palas Kafi (dhun)

Rāga Bilaskhani Todi

Tablā: Kanai Dutt

Gramco India, LP EALP 1251 (1960)

Rāga Shree

Rāga Sindhi Bhairavi

Tablā: Alla Rakha

Gramco India, LP EASD 1296 (1963)

«Concert for Bangla Desh»

Rāga Bangla Dhun

Tablā: Alla Rakha

Apple, 3LP, face A, STCX 3385 (1971)

«In Concert 1972»

Rāga Hem Bihag

Rāga Manj Khammaj

Rāga Sindhi Bhairavi

Tablā: Alla Rakha

Apple/Gramco India 2LP SAPDO 1002 (1972)

«Jugalbandi »

Rāga Khammaj

Rāga Durga

Tablā: Alla Rakha

Gramco India, LP ECSD 41516 (1983)

Rāga Mishra Pilu

Angel/Gramco India, LP DS 37920, CD DS 37920 (1983) 


\section{Duoa avec Nikhil Banerjee}

Rāga Manj Khammaj

Rāga Misra Mand

Tablā: Mahapurush Misra

Connoisseur Society LP CS 2055 (1973)

«Maihar Festival»

(avec la participation de Dhyanesh Khan: sarod)

Rāga Malayam Smruti

Rāga Bhairavi

Tablā: Kishan Maharaj

Montage, MC G 30518 (1978)

\section{Duos avec L. Subramaniam}

«India's Master Musicians»

Rāga Sindhi Bhairavi

Tablā: Zakir Hussain

Mridangam: Ramnad V. Raghavan

Bainbridge, LP RSD 27, Ravi Shankar Music

Circle, MC RSMC 27, CD RSMC D-103 (1981)

«Ethereal Duet»

Rāga Jog

Tablā: Zakir Hussain

Mridangam: Ramnad V. Raghavan

Ganesh Records, LP DRLS 4002 (1982)

\section{Rencontres Est-Ouest}

«The Flowers of Evil»

Six poèmes traduits de Beaudelaire, récités en anglais par Yvette Mimieux, environnement sonore de sarod et tablā. Tablā: Mahapurush Mishra

Connoisseur Society, LP CS 2007 (1968)
«Karuna Supreme»

Saxophone: John Handy

Tablā: Zakir Hussain

BASF 22791 (1974)

«Rainbow»

Saxophone: John Handy

Violon: L. Subramaniam

Tablā: Shyam Kane

MPS/VERVE 821.666-1 (1980)

«Journey»

Tablā: Swapan Chaudhuri

Dholak: Pranesh Khan

Claviers, guitare: Jai Uttal Percussions: John

Bergamo Sitār: James Pomerantz

Basse: Brian Godden

Triloka Records (1990)

\section{Disques d'Allauddin Khan}

Rāga Lalit (sarod)
Rāga Jila
Rāga Tilak Kamod
Rāga Bihag
Rāga Bhairavi
Rāga Ghara
Rāga Sindhura (violon)
Rāga Bihag
Rāga Manj Khammaj
Rāga Maljunga
Kirtan
Kirtan
Gramco India, LP
(réédition de disques 78t)
Rāga Kaushi Bhairav
Rāga Hem
Gramco India, LP ECLP 2757 (1976)

\section{Sources}

«A Discography of Hindustani and Karnatic Music», de Michael S. Kinnear (London, Greenwood Press, 1984)

Catalogues et fiches d'édition de: Gramophone Company of India, Ltd., Alam Madina Productions, Ravi Shankar Music Circle et Chhanda Dhara

Disques de différentes collections privée. 